\title{
Weed Control, Environmental Impact, and Net-Profit of Two-Pass Weed Management Strategies in Dicamba-Resistant Soybean (Glycine max) Using Conventional Tillage
}

\author{
Matthew G. Underwood, Nader Soltan*, David C. Hooker, Darren E. Robinson, Joseph P. Vink, \\ Clarence J. Swanton, Peter H. Sikkema \\ Department of Plant Agriculture, University of Guelph, Ridgetown, Canada \\ Email: *soltanin@uoguelph.ca
}

How to cite this paper: Underwood, M.G., Soltan, N., Hooker, D.C., Robinson, D.E., Vink, J.P., Swanton, C.J. and Sikkema, P.H. (2017) Weed Control, Environmental Impact, and Net-Profit of Two-Pass Weed Management Strategies in Dicamba-Resistant Soybean (Glycine max) Using Conventional Tillage. American Journal of Plant Sciences, 8, 3414-3428.

https://doi.org/10.4236/ajps.2017.813229

Received: November 15, 2017

Accepted: December 18, 2017

Published: December 21, 2017

Copyright $\odot 2017$ by authors and Scientific Research Publishing Inc. This work is licensed under the Creative Commons Attribution International License (CC BY 4.0).

http://creativecommons.org/licenses/by/4.0/

\begin{abstract}
Traditional two-pass weed management strategies need to be compared with new strategies in glyphosate/dicamba-resistant soybean. Weed control, soybean yield, partial profitability and environmental impact (EI) were evaluated in glyphosate/dicamba-resistant soybean using dicamba applied alone or in a tank-mix with dimethenamid-P applied preemergence (PRE). Trials were conducted at three locations during 2014 and 2015. Several PRE herbicides provided excellent control of broadleaf and grass weeds. Dicamba provided > $91 \%$ control of broadleaf weeds, and the addition of dimethenamid-P improved grass control. All weed species at the trial locations were controlled > 94\% following a postemergence (POST) application of glyphosate. Weed interference reduced soybean seed yield $33 \%$ where no herbicide was applied. A single POST application of glyphosate had the lowest EI. Several treatments improved early-season weed control and reduced early-season weed density and biomass compared to glyphosate and had similar EI values. In this study, there was no benefit to yield or partial profit by including a PRE herbicide for weed management; however, the inclusion of multiple modes-of-action in a herbicide program may reduce the selection for herbicide-resistant weeds.
\end{abstract}

\section{Keywords}

Density, Environmental Impact, Injury, Profit Analysis, Tillage,

Yield, Weed Control

\section{Introduction}

Soybean has been grown in Ontario, Canada since 1881 [1] and it is currently 
the largest and most valuable cash crop in the province. In 2015, soybean was seeded on 1.2 million hectares, and produced over 3.6 million tonnes, which was worth close to $\$ 1.6$ billion [2]. Weed control is an important component of soybean production, especially early in the growing season [3]. Weed interference can reduce soybean yield by as much as $80 \%$ [4]. Soybean is most susceptible to yield loss during the critical weed-free period (CWFP) that begins at V1 and ends at V4 [5] [6] [7], which is the reason why herbicide applications are typically made prior to soybean emergence and soybeans are maintained weed-free through the end of the vegetative growth period prior to flowering.

Weed control was greatly simplified when glyphosate-resistant (GR) soybean was commercialized in Ontario in 1997. Glyphosate is a Group 9, non-selective herbicide that controls over 300 weed species [8]. It has a wide margin of crop safety, low toxicity, and a flexible application window [6] [9] [10]; these factors contributed to the high adoption rate of GR soybean. In 2015, 70\% of Ontario soybean was produced from GR cultivars. While glyphosate applied alone may be a simple and effective weed management strategy, its lack of residual control often requires glyphosate to be applied multiple times in a growing season to obtain full-season weed control [11] [12] [13]. Furthermore, the sequential application of a single herbicide with one mode-of-action may select for herbicide-resistant (HR) weed biotypes; for this reason, the repeated use of a single mode-of-action is strongly discouraged [13]. The growing concern of selecting HR weed biotypes has prompted research for weed management solutions that can fill the agronomic gaps left by using only glyphosate.

Two-pass weed management strategies including a pre-plant (PP) or pre-emergence (PRE) residual herbicide followed by a postemergence (POST) herbicide often result in full-season weed control using multiple modes-of-action [11] [14]. The addition of multiple modes-of-action and including multiple application timings can improve weed control, minimize soybean yield loss caused by weed interference, and reduce selection pressure for HR weed biotypes [13] [15] [16].

Soybean resistant to both dicamba and glyphosate (DR soybean) will be commercially available for the 2017 growing season. Dicamba is a Group 4, systemic broadleaf herbicide that controls over 40 broadleaf weed species [17] [18] and provides short-term residual weed control [19]. Dicamba provides excellent control of many troublesome weeds such as common and giant ragweed ( $\mathrm{Am}$ brosia artemisiifolia L., Ambrosia trifida L.), common lambsquarters (Chenopodium album L.), and redroot pigweed (Amaranthus retroflexus L.) [20]. In addition, dicamba controls several GR broadleaf weed species found in Canada and the United States. Dicamba (600 g.ai.ha ${ }^{-1}$ ) applied PP or POST controls GR Canada fleabane (Conyza canadensis L. Cronq.), common ragweed, giant ragweed, waterhemp (Amaranthus tuberculatus var. rudis J.D. Sauer) and Palmer amaranth (Amaranthus palmeri S. Wats.) [21] [22] [23] [24].

It is important to design effective weed management strategies for controlling weeds, but with minimal impact on the environment. Brookes and Barfoot [25] 
and Gianessi [26] noted that the agricultural industry is being pressured to reduce the environmental impact (EI) of production practices. Thus, producers should consider the EI of their herbicide program in addition to effective weed control. The EI can be reduced by reducing herbicide rates or applying herbicides that have a lower EI; the environmental impact quotient (EIQ) can help determine the relative risk of various herbicide programs. The EIQ is a calculated potential risk based on physiochemical and toxicological properties of a herbicide [12] [14] [27] [28]. Toxicological data are gathered on the potential risk to the farm worker, consumer, and ecosystem, and include variables such as acute and chronic toxicity, toxicity to birds, aquatic species, and beneficial insects, as well as soil half-life, leaching potential, and the half-life of the chemical within the plant [29]. The EIQ is then multiplied by the application rate to determine the EI. Pesticides with higher EI values are deemed to pose a greater environmental risk.

Farmers must ensure they are producing an economically sustainable crop in addition to herbicide efficacy, herbicide cost, crop safety, and EI. The commercial release of DR soybean provides additional weed control strategies that need to be compared with traditional two-pass weed management strategies in soybean. The objective of this study was to evaluate herbicide programs for their crop safety, weed control efficacy, profitability, and EI in DR soybean produced with conventional tillage.

\section{Materials and Methods}

Field trials were conducted over a two-year period $(2014,2015)$ at three sites in southwestern Ontario, Canada. The experiments were located at the University of Guelph-Ridgetown Campus, Ridgetown, Ontario; the Huron Research Station, Exeter, Ontario; and the Woodstock Research Station, Woodstock, Ontario. Location, year, seeding date, and herbicide application dates are presented in Table 1. Seedbed preparation for all sites included moldboard plowing in the autumn, followed by two passes with a field cultivator with rolling basket harrows in the spring.

The trials were established in a randomized complete block design (RCBD) with four replications. Treatments are listed in Tables 2-7. Treatments included a weedy (non-treated) and weed-free controls. Weed-free plots were maintained weed-free by applying a tank-mix of imazethapyr $\left(100 \mathrm{~g} \cdot \mathrm{ai} \cdot \mathrm{ha}^{-1}\right)$ plus metribuzin (400 g.ai $\cdot \mathrm{ha}^{-1}$ ) PRE and additional POST applications of glyphosate (900 g.ae.ha ${ }^{-1}$ ) were made as required. Glyphosate/dicamba-resistant soybean (Roundup Ready $2 \mathrm{Xtend}^{\mathrm{TM}}$ ) was seeded at 400,000 seeds ha $^{-1}$ in plots that were $3.0 \mathrm{~m}$ wide (4 rows spaced $0.75 \mathrm{~m}$ apart) and 8 and $10 \mathrm{~m}$ long in Ridgetown and Exeter, respectively, and 5.75 and $8 \mathrm{~m}$ long in Woodstock in 2014 and 2015, respectively.

Herbicides were applied with a $\mathrm{CO}_{2}$-pressurized backpack sprayer calibrated to deliver $200 \mathrm{~L} \cdot \mathrm{ha}^{-1}$ of spray solution at $210 \mathrm{kPa}$. A $1.5 \mathrm{~m}$ spray boom was used equipped with 4 ultra-low drift nozzles (Hypro ULD 120-02, New Brighton, MN, 
Table 1. Location, year, seeding date and herbicide application timing and date.

\begin{tabular}{|c|c|c|c|c|}
\hline Environment & Year & Seeding Date & Application timing ${ }^{a}$ & Herbicide application Date \\
\hline \multirow{4}{*}{ Ridgetown } & \multirow{2}{*}{2014} & \multirow{2}{*}{ June 04} & $\mathrm{PP}$ & June 04 \\
\hline & & & POST & July 02 \\
\hline & \multirow{2}{*}{2015} & \multirow{2}{*}{ May 20} & $\mathrm{PP}$ & May 21 \\
\hline & & & POST & June 25 \\
\hline \multirow{4}{*}{ Exeter } & \multirow{2}{*}{2014} & \multirow{2}{*}{ June 01} & PP & June 05 \\
\hline & & & POST & July 05 \\
\hline & \multirow{2}{*}{2015} & \multirow{2}{*}{ May 08} & PP & May 09 \\
\hline & & & POST & June 19 \\
\hline \multirow{4}{*}{ Woodstock } & \multirow{2}{*}{2014} & \multirow{2}{*}{ June 05} & PP & June 06 \\
\hline & & & POST & July 04 \\
\hline & \multirow{2}{*}{2015} & \multirow{2}{*}{ May 13} & PP & May 14 \\
\hline & & & POST & June 16 \\
\hline
\end{tabular}

${ }^{a} \mathrm{PP}$ application timing represents Pre-Plant and POST represents Post-Emergence.

Table 2. Control of redroot pigweed (AMARE), common ragweed (AMBEL), common lambsquarters (CHEAL), lady's thumb (POLPE), and green foxtail (SETVI) 3 weeks after soybean emergence (WAE) in response to a preemergence (PRE) herbicide application to conventional till soybean averaged across three locations in 2014 and 2015.

\begin{tabular}{ccccccc}
\hline Treatment & Rate & AMARE $^{\mathrm{c}}$ & AMBEL & CHEAL & POLPE & SETVI \\
\cline { 2 - 7 } & g.ai-ha ${ }^{-1}$ & & & $\%$ & & \\
\hline Weed-Free Control & & $100 \mathrm{a}$ & $100 \mathrm{a}$ & $100 \mathrm{a}$ & $100 \mathrm{a}$ & $100 \mathrm{a}$ \\
Glyphosate & 900 & $0 \mathrm{e}$ & $0 \mathrm{~g}$ & $0 \mathrm{e}$ & $0 \mathrm{f}$ & $0 \mathrm{~h}$ \\
Imazethapyr & 100 & $96 \mathrm{c}$ & $79 \mathrm{de}$ & $90 \mathrm{bc}$ & $99 \mathrm{abc}$ & $79 \mathrm{cde}$ \\
Imazethapyr/saflufenacil & 100 & $97 \mathrm{abc}$ & $77 \mathrm{de}$ & $86 \mathrm{~cd}$ & $98 \mathrm{abcd}$ & $69 \mathrm{ef}$ \\
Saflufenacil/dimethenamid-p & 245 & $82 \mathrm{~d}$ & $57 \mathrm{f}$ & $65 \mathrm{~d}$ & $76 \mathrm{e}$ & $57 \mathrm{f}$ \\
Imazethapyr & 75 & & & & & \\
Metribuzin & 425 & $99 \mathrm{abc}$ & $91 \mathrm{bcd}$ & $97 \mathrm{abc}$ & $99 \mathrm{ab}$ & $91 \mathrm{~b}$ \\
Chlorimuron & 9 & & & & & \\
Metribuzin & 412 & $98 \mathrm{abc}$ & $91 \mathrm{bcd}$ & $94 \mathrm{abc}$ & $76 \mathrm{e}$ & $76 \mathrm{de}$ \\
Chlorimuron & 9 & & & & & \\
Imazethapyr & 75 & $98 \mathrm{abc}$ & $87 \mathrm{~cd}$ & $93 \mathrm{bc}$ & $99 \mathrm{ab}$ & $90 \mathrm{bc}$ \\
Pyroxasulfone & 100 & & & & & \\
Sulfentrazone & 100 & $98 \mathrm{abc}$ & $67 \mathrm{ef}$ & $95 \mathrm{abc}$ & $98 \mathrm{abcd}$ & $87 \mathrm{bcd}$ \\
Dicamba & 600 & $97 \mathrm{bc}$ & $99 \mathrm{ab}$ & $92 \mathrm{bc}$ & $99 \mathrm{ab}$ & $20 \mathrm{~g}$ \\
Dimethenamid-p & 544 & $98 \mathrm{abc}$ & $89 \mathrm{~cd}$ & $88 \mathrm{c}$ & $85 \mathrm{e}$ & $90 \mathrm{bc}$ \\
Dicamba & 300 & & & & & \\
Metribuzin & 413 & & & & & \\
Imazethapyr & 77 & $100 \mathrm{ab}$ & $98 \mathrm{ab}$ & $99 \mathrm{ab}$ & $99 \mathrm{ab}$ & $95 \mathrm{~b}$ \\
Flumioxazin & 96 & & & & & \\
s-metolachlor/metribuzin & 1443 & $97 \mathrm{bc}$ & $82 \mathrm{cde}$ & $89 \mathrm{c}$ & $90 \mathrm{cde}$ & $95 \mathrm{~b}$ \\
Pyroxasulfone/flumioxazin & 160 & $100 \mathrm{abc}$ & $94 \mathrm{bc}$ & $94 \mathrm{abc}$ & $88 \mathrm{de}$ & $92 \mathrm{~b}$ \\
\hline
\end{tabular}

Means followed by the same letter within a column are not significantly different according to Fisher's pro-

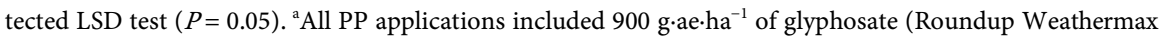
540SL formulation); ${ }^{\mathrm{b}}$ All treatments received a POST application of $900 \mathrm{~g} \cdot \mathrm{ae} \cdot \mathrm{ha}^{-1}$ of glyphosate (Roundup Weathermax 540SL formulation) at the V4 soybean growth stage; 'All data have been pooled across locations and years. Data presented in the table have been backtransformed to the original scale. 
Table 3. Density of redroot pigweed (AMARE), common ragweed (AMBEL), common lambsquarters (CHEAL), lady's thumb (POLPE), barnyard grass (ECHCG) and green foxtail (SETVI) evaluated 3 weeks after soybean emergence (WAE) in response to a preemergence (PRE) herbicide application to conventional till soybean ground averaged across three locations across 2014 and 2015.

\begin{tabular}{|c|c|c|c|c|c|c|c|}
\hline \multirow{2}{*}{ Treatment $^{\mathrm{ab}}$} & Rate & AMARE $^{c}$ & AMBEL & CHEAL & POLPE & ECHCG & SETVI \\
\hline & g.ai.ha ${ }^{-1}$ & \multicolumn{6}{|c|}{ plants $\mathrm{m}^{-2}$} \\
\hline Weedy Control & & $12 \mathrm{a}$ & $8 \mathrm{a}$ & $26 \mathrm{a}$ & $6 a$ & $17 \mathrm{a}$ & $56 a$ \\
\hline Glyphosate & 900 & $10 \mathrm{a}$ & $8 \mathrm{ab}$ & $16 \mathrm{ab}$ & $6 a$ & $19 \mathrm{a}$ & $34 \mathrm{ab}$ \\
\hline Imazethapyr & 100 & $1 b$ & $6 a b c$ & $4 \mathrm{cde}$ & $1 b c d$ & $16 \mathrm{a}$ & 5 defg \\
\hline Imazethapyr/saflufenacil & 100 & obcd & $4 \mathrm{~cd}$ & $3 \mathrm{def}$ & $1 \mathrm{~cd}$ & $13 a b c$ & $7 \mathrm{def}$ \\
\hline Saflufenacil/dimethenamid-p & 245 & $1 \mathrm{bc}$ & $4 \mathrm{~cd}$ & $8 \mathrm{bc}$ & $2 \mathrm{~b}$ & $9 \mathrm{abcd}$ & $11 \mathrm{~cd}$ \\
\hline Imazethapyr & 75 & \multirow{2}{*}{ obcd } & \multirow{2}{*}{$3 c d e$} & \multirow{2}{*}{$1 \mathrm{fgh}$} & \multirow{2}{*}{$0 \mathrm{~d}$} & \multirow{2}{*}{$11 \mathrm{abc}$} & \multirow{2}{*}{ 4efgh } \\
\hline Metribuzin & 425 & & & & & & \\
\hline Chlorimuron & 9 & \multirow{2}{*}{ obcd } & \multirow{2}{*}{$2 \mathrm{de}$} & \multirow{2}{*}{2 efgh } & \multirow{2}{*}{$1 b c d$} & \multirow{2}{*}{$14 \mathrm{ab}$} & \multirow{2}{*}{$9 \mathrm{cde}$} \\
\hline Metribuzin & 412 & & & & & & \\
\hline Chlorimuron & 9 & \multirow{2}{*}{ Obcd } & \multirow{2}{*}{$5 \mathrm{abcd}$} & \multirow{2}{*}{$4 c d e$} & \multirow{2}{*}{$0 \mathrm{~d}$} & \multirow{2}{*}{$10 \mathrm{abc}$} & \multirow{2}{*}{$5 \mathrm{defg}$} \\
\hline Imazethapyr & 75 & & & & & & \\
\hline Pyroxasulfone & 100 & \multirow{2}{*}{ Obcd } & \multirow{2}{*}{$5 \mathrm{abcd}$} & \multirow{2}{*}{ 1gh } & \multirow{2}{*}{$2 b c$} & \multirow{2}{*}{$6 \mathrm{cde}$} & \multirow{2}{*}{4 efgh } \\
\hline Sulfentrazone & 100 & & & & & & \\
\hline Dicamba & 600 & $1 b$ & lef & 3defg & $2 \mathrm{~b}$ & $19 \mathrm{a}$ & $23 \mathrm{bc}$ \\
\hline Dimethenamid-p & 544 & \multirow{2}{*}{$1 b c d$} & \multirow{2}{*}{$4 \mathrm{bcd}$} & \multirow{2}{*}{$6 \mathrm{~cd}$} & \multirow{2}{*}{$2 b c$} & \multirow{2}{*}{$4 \mathrm{de}$} & \multirow{2}{*}{3 fgh } \\
\hline Dicamba & 300 & & & & & & \\
\hline Metribuzin & 413 & \multirow{3}{*}{$0 \mathrm{~cd}$} & \multirow{3}{*}{ 1f } & & & & \\
\hline Imazethapyr & 77 & & & $\mathrm{Oh}$ & $0 \mathrm{~d}$ & $7 \mathrm{bcde}$ & $2 \mathrm{gh}$ \\
\hline Flumioxazin & 96 & & & & & & \\
\hline s-metolachlor/metribuzin & 1443 & $1 \mathrm{bcd}$ & $5 \mathrm{abcd}$ & $3 \mathrm{def}$ & lbcd & $4 \mathrm{e}$ & $1 \mathrm{~h}$ \\
\hline Pyroxasulfone/flumioxazin & 160 & $0 \mathrm{~d}$ & If & $1 \mathrm{gh}$ & $1 \mathrm{~cd}$ & $4 \mathrm{de}$ & $2 \mathrm{gh}$ \\
\hline
\end{tabular}

Means followed by the same letter within a column are not significantly different according to Fisher's protected LSD test $(P=0.05)$. ${ }^{\text {all }}$ PP applications included $900 \mathrm{~g} \cdot \mathrm{ai}^{\mathrm{h}} \mathrm{ha}^{-1}$ of glyphosate (Roundup Weathermax 540SL formulation); ${ }^{b}$ All treatments received a POST application of $900 \mathrm{~g} \cdot \mathrm{ae} \cdot \mathrm{ha}^{-1}$ of glyphosate (Roundup Weathermax 540SL formulation) at the V4 soybean growth stage; 'All data have been pooled across locations and years. Data presented in the table have been backtransformed to the original scale.

Table 4. Dry weight of redroot pigweed (AMARE), common ragweed (AMBEL), common lambsquarters (CHEAL), lady's thumb (POLPE), barnyardgrass (ECHCG), and green foxtail (SETVI) evaluated 3 weeks after soybean emergence (WAE) in response to a preemergence (PRE) herbicide application to conventional till soybean ground averaged across three locations over 2014 and 2015.

\begin{tabular}{|c|c|c|c|c|c|c|c|}
\hline \multirow{2}{*}{ Treatment $^{\mathrm{ab}}$} & Rate & AMARE $^{c}$ & AMBEL & CHEAL & POLPE & ECHCG & SETVI \\
\hline & g.ai $\cdot \mathrm{ha}^{-1}$ & \multicolumn{6}{|c|}{ plants $\mathrm{m}^{-2}$} \\
\hline Weedy Control & & $2.0 \mathrm{a}$ & $2.1 \mathrm{a}$ & $2.5 \mathrm{a}$ & $1.2 \mathrm{a}$ & $3.5 \mathrm{a}$ & $5.2 \mathrm{a}$ \\
\hline Glyphosate & 900 & $2.0 \mathrm{a}$ & $2.1 \mathrm{a}$ & $2.4 \mathrm{a}$ & $1.0 \mathrm{ab}$ & $3.8 \mathrm{a}$ & $5.2 \mathrm{a}$ \\
\hline Imazethapyr & 100 & $0.3 b$ & $1.3 \mathrm{ab}$ & $0.8 \mathrm{bc}$ & $0.2 \mathrm{def}$ & $1.9 \mathrm{~b}$ & $0.9 \mathrm{cde}$ \\
\hline Imazethapyr/saflufenacil & 100 & $0.1 b$ & $0.8 \mathrm{bcde}$ & $0.8 \mathrm{bc}$ & $0.2 \mathrm{def}$ & $1.4 \mathrm{bcd}$ & $0.9 \mathrm{~cd}$ \\
\hline Saflufenacil/dimethenamid-p & 245 & $0.4 b$ & $1.0 \mathrm{bcd}$ & $1.9 \mathrm{ab}$ & $0.7 \mathrm{abc}$ & $1.9 \mathrm{~b}$ & $1.3 \mathrm{c}$ \\
\hline Imazethapyr & 425 & $0.1 b$ & $0.6 \mathrm{bcdef}$ & $0.2 \mathrm{~cd}$ & $0.1 \mathrm{f}$ & $1.2 \mathrm{bcd}$ & $0.5 \mathrm{def}$ \\
\hline $\begin{array}{l}\text { Chlorimuron } \\
\text { Metribuzin }\end{array}$ & $\begin{array}{c}9 \\
412\end{array}$ & $0.2 \mathrm{~b}$ & $0.4 \mathrm{def}$ & $0.4 \mathrm{~cd}$ & $0.2 \mathrm{def}$ & $1.6 \mathrm{bc}$ & $0.8 \mathrm{cdef}$ \\
\hline
\end{tabular}


M. G. Underwood et al.

\section{Continued}

\begin{tabular}{ccccccccc}
\hline Chlorimuron & 9 & & & & & & & \\
Imazethapyr & 75 & $0.1 \mathrm{~b}$ & $0.8 \mathrm{bcde}$ & $0.6 \mathrm{~cd}$ & $0.1 \mathrm{f}$ & $1.2 \mathrm{bcd}$ & $0.7 \mathrm{cdef}$ \\
Pyroxasulfone & 100 & & & & & & \\
Sulfentrazone & 100 & $0.1 \mathrm{~b}$ & $1.0 \mathrm{bcd}$ & $0.2 \mathrm{~cd}$ & $0.5 \mathrm{bcd}$ & $1.3 \mathrm{bcd}$ & $0.7 \mathrm{cdef}$ \\
Dicamba & 600 & $0.1 \mathrm{~b}$ & $0.2 \mathrm{ef}$ & $0.1 \mathrm{~cd}$ & $0.3 \mathrm{cdef}$ & $3.8 \mathrm{a}$ & $2.8 \mathrm{~b}$ \\
Dimethenamid-p & 544 & $0.1 \mathrm{~b}$ & $0.5 \mathrm{cdef}$ & $0.5 \mathrm{~cd}$ & $0.5 \mathrm{bcde}$ & $0.7 \mathrm{~d}$ & $0.4 \mathrm{def}$ \\
Dicamba & 300 & & & & & & \\
Metribuzin & 413 & & & & & & \\
Imazethapyr & 77 & $0 \mathrm{~b}$ & $0.1 \mathrm{f}$ & $0 \mathrm{~d}$ & $0 \mathrm{f}$ & $1.0 \mathrm{bcd}$ & $0.2 \mathrm{f}$ \\
Flumioxazin & 96 & & & & & & \\
s-metolachlor/metribuzin & 1443 & $0.1 \mathrm{~b}$ & $1.0 \mathrm{bc}$ & $0.8 \mathrm{bc}$ & $0.2 \mathrm{cdef}$ & $0.7 \mathrm{~d}$ & $0.3 \mathrm{ef}$ \\
Pyroxasulfone/flumioxazin & 160 & $0 \mathrm{~b}$ & $0.1 \mathrm{f}$ & $0.3 \mathrm{~cd}$ & $0.1 \mathrm{ef}$ & $0.8 \mathrm{~cd}$ & $0.3 \mathrm{def}$ \\
\hline
\end{tabular}

Means followed by the same letter within a column are not significantly different according to Fisher's pro-

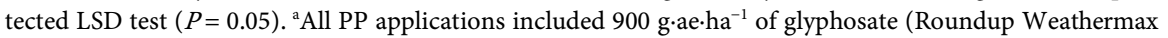
540SL formulation); ${ }^{b}$ All treatments received a POST application of $900 \mathrm{~g} \cdot \mathrm{ae}^{\mathrm{h}} \mathrm{ha}^{-1}$ of glyphosate (Roundup Weathermax 540SL formulation) at the V4 soybean growth stage; ${ }^{\mathrm{c} A l l}$ data have been pooled across locations and years. Data presented in the table have been backtransformed to the original scale.

Table 5. Control of redroot pigweed (AMARE), common ragweed (AMBEL), common lambsquarters (CHEAL), lady's thumb (POLPE), barnyardgrass (ECHCG), and green foxtail (SETVI) evaluated 4 weeks after the POST application (WAA) in response to a preemergence (PRE) herbicide application to conventional till soybean ground followed by a POST application of glyphosate averaged across three locations over 2014 and 2015.

\begin{tabular}{|c|c|c|c|c|c|c|c|}
\hline \multirow{2}{*}{ Treatment $^{\mathrm{ab}}$} & Rate & $\mathrm{AMARE}^{\mathrm{c}}$ & AMBEL & CHEAL & POLPE & ECHCG & SETVI \\
\hline & g.ai.ha ${ }^{-1}$ & \multicolumn{6}{|c|}{ plants $\mathrm{m}^{-2}$} \\
\hline Weed-Free Control & & $100 \mathrm{a}$ & $100 \mathrm{a}$ & $100 \mathrm{a}$ & $100 \mathrm{a}$ & $100 \mathrm{a}$ & $100 \mathrm{a}$ \\
\hline Glyphosate & 900 & $96 \mathrm{~d}$ & $97 \mathrm{e}$ & $96 \mathrm{e}$ & $96 \mathrm{bcd}$ & $97 \mathrm{de}$ & $97 \mathrm{de}$ \\
\hline Imazethapyr & 100 & $99 \mathrm{abc}$ & $98 \mathrm{de}$ & $99 \mathrm{abcd}$ & $100 \mathrm{ab}$ & $97 \mathrm{cde}$ & $98 \mathrm{bcd}$ \\
\hline Imazethapyr/saflufenacil & 100 & $99 \mathrm{abc}$ & $99 \mathrm{cde}$ & $99 \mathrm{abcd}$ & 99abcd & $98 \mathrm{bcd}$ & $99 \mathrm{bc}$ \\
\hline Saflufenacil/dimethenamid-p & 245 & $98 \mathrm{bcd}$ & $98 \mathrm{de}$ & 98de & $96 \mathrm{bcd}$ & $96 \mathrm{de}$ & $98 \mathrm{cde}$ \\
\hline Imazethapyr & 75 & \multirow{2}{*}{$100 \mathrm{ab}$} & \multirow{2}{*}{$99 \mathrm{abc}$} & \multirow{2}{*}{$100 \mathrm{ab}$} & \multirow{2}{*}{$100 \mathrm{ab}$} & \multirow{2}{*}{$97 \mathrm{cde}$} & \multirow{2}{*}{$98 \mathrm{bcde}$} \\
\hline Metribuzin & 425 & & & & & & \\
\hline Chlorimuron & 9 & \multirow{2}{*}{$100 \mathrm{ab}$} & \multirow{2}{*}{$99 \mathrm{bc}$} & \multirow{2}{*}{$100 \mathrm{ab}$} & \multirow{2}{*}{ 99abcd } & \multirow{2}{*}{$98 \mathrm{bcde}$} & \multirow{2}{*}{$98 \mathrm{bcde}$} \\
\hline Metribuzin & 412 & & & & & & \\
\hline Chlorimuron & 9 & \multirow{2}{*}{$100 \mathrm{ab}$} & \multirow{2}{*}{$99 \mathrm{bcd}$} & \multirow{2}{*}{$100 \mathrm{abc}$} & \multirow{2}{*}{$100 \mathrm{a}$} & \multirow{2}{*}{$97 \mathrm{cde}$} & \multirow{2}{*}{$99 \mathrm{bcd}$} \\
\hline Imazethapyr & 75 & & & & & & \\
\hline Pyroxasulfone & 100 & \multirow{2}{*}{$100 \mathrm{ab}$} & \multirow{2}{*}{$99 \mathrm{bcd}$} & \multirow{2}{*}{$100 \mathrm{ab}$} & \multirow{2}{*}{$95 \mathrm{~d}$} & \multirow{2}{*}{$99 \mathrm{abc}$} & \multirow{2}{*}{$99 b$} \\
\hline Sulfentrazone & 100 & & & & & & \\
\hline Dicamba & 600 & $96 \mathrm{~cd}$ & $99 \mathrm{bcd}$ & $98 \mathrm{de}$ & $98 \mathrm{abcd}$ & $95 \mathrm{e}$ & $97 \mathrm{e}$ \\
\hline Dimethenamid-p & 544 & \multirow{2}{*}{$98 \mathrm{bcd}$} & \multirow{2}{*}{$99 \mathrm{bcd}$} & \multirow{2}{*}{$98 \mathrm{cde}$} & \multirow{2}{*}{$95 \mathrm{~cd}$} & \multirow{2}{*}{$97 \mathrm{de}$} & \multirow{2}{*}{$98 \mathrm{bcde}$} \\
\hline Dicamba & 300 & & & & & & \\
\hline Metribuzin & 413 & \multirow{3}{*}{$100 \mathrm{a}$} & \multirow{3}{*}{$100 \mathrm{ab}$} & & & & \\
\hline Imazethapyr & 77 & & & $100 \mathrm{a}$ & $100 \mathrm{a}$ & 97de & $98 \mathrm{bcde}$ \\
\hline Flumioxazin & 96 & & & & & & \\
\hline s-metolachlor/metribuzin & 1443 & 99abcd & $99 \mathrm{cde}$ & $98 \mathrm{bcde}$ & $98 \mathrm{abcd}$ & $100 \mathrm{ab}$ & $99 \mathrm{~b}$ \\
\hline Pyroxasulfone/flumioxazin & 160 & $100 \mathrm{ab}$ & $100 \mathrm{abc}$ & $100 \mathrm{ab}$ & $99 \mathrm{abc}$ & 98bcde & $99 \mathrm{bc}$ \\
\hline
\end{tabular}

Means followed by the same letter within a column are not significantly different according to Fisher's protected LSD test $(P=0.05) .{ }^{a}$ All PP applications included $900 \mathrm{~g} \cdot \mathrm{ae} \cdot \mathrm{ha}^{-1}$ of glyphosate (Roundup Weathermax

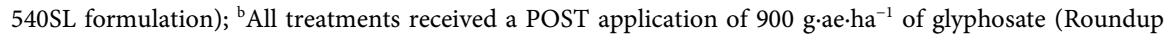
Weathermax 540SL formulation) at the V4 soybean growth stage; ${ }^{\mathrm{C}}$ All data have been pooled across locations and years. Data presented in the table have been backtransformed to the original scale. 
Table 6. Control of redroot pigweed (AMARE), common ragweed (AMBEL), common lambsquarters (CHEAL), lady's thumb (POLPE), barnyardgrass (ECHCG), and green foxtail (SETVI) evaluated 8 weeks after the POST application (WAA) in response to a preemergence (PRE) herbicide application to conventional till soybean ground followed by a POST application of glyphosate averaged across three locations over 2014 and 2015.

\begin{tabular}{|c|c|c|c|c|c|c|c|}
\hline \multirow{2}{*}{ Treatment $^{\mathrm{ab}}$} & Rate & AMARE $^{\mathrm{c}}$ & AMBEL & CHEAL & POLPE & ECHCG & SETVI \\
\hline & $\mathrm{g} \cdot \mathrm{ai} \mathrm{ha}^{-1}$ & \multicolumn{6}{|c|}{ plants $\mathrm{m}^{-2}$} \\
\hline Weed-Free Control & & $100 \mathrm{a}$ & $100 \mathrm{a}$ & $100 \mathrm{a}$ & $100 \mathrm{a}$ & $100 \mathrm{a}$ & $100 \mathrm{a}$ \\
\hline Glyphosate & 900 & $96 d$ & $97 \mathrm{e}$ & $96 \mathrm{e}$ & $96 \mathrm{bcd}$ & $97 \mathrm{de}$ & $97 \mathrm{de}$ \\
\hline Imazethapyr & 100 & $99 \mathrm{abc}$ & 98de & 99abcd & $100 \mathrm{ab}$ & $97 \mathrm{cde}$ & $98 \mathrm{bcd}$ \\
\hline Imazethapyr/saflufenacil & 100 & $99 \mathrm{abc}$ & $99 \mathrm{cde}$ & 99abcd & 99abcd & $98 \mathrm{bcd}$ & $99 \mathrm{bc}$ \\
\hline Saflufenacil/dimethenamid-p & 245 & $98 \mathrm{bcd}$ & 98de & 98de & $96 \mathrm{bcd}$ & 96de & $98 \mathrm{cde}$ \\
\hline $\begin{array}{l}\text { Imazethapyr } \\
\text { Metribuzin }\end{array}$ & 425 & $100 \mathrm{ab}$ & $99 \mathrm{abc}$ & $100 \mathrm{ab}$ & $100 \mathrm{ab}$ & $97 \mathrm{cde}$ & $98 \mathrm{bcde}$ \\
\hline $\begin{array}{l}\text { Chlorimuron } \\
\text { Metribuzin }\end{array}$ & $\begin{array}{c}9 \\
412\end{array}$ & $100 \mathrm{ab}$ & $99 \mathrm{bc}$ & $100 \mathrm{ab}$ & 99abcd & $98 \mathrm{bcde}$ & $98 \mathrm{bcde}$ \\
\hline $\begin{array}{l}\text { Chlorimuron } \\
\text { Imazethapyr }\end{array}$ & 75 & $100 \mathrm{ab}$ & $99 \mathrm{bcd}$ & $100 \mathrm{abc}$ & $100 \mathrm{a}$ & $97 \mathrm{cde}$ & $99 \mathrm{bcd}$ \\
\hline $\begin{array}{l}\text { Pyroxasulfone } \\
\text { Sulfentrazone }\end{array}$ & 100 & $100 \mathrm{ab}$ & $99 \mathrm{bcd}$ & $100 \mathrm{ab}$ & $95 d$ & $99 \mathrm{abc}$ & $99 b$ \\
\hline Dicamba & 600 & $96 \mathrm{~cd}$ & $99 \mathrm{bcd}$ & $98 \mathrm{de}$ & 98abcd & $95 \mathrm{e}$ & $97 \mathrm{e}$ \\
\hline $\begin{array}{c}\text { Dimethenamid-p } \\
\text { Dicamba }\end{array}$ & $\begin{array}{l}544 \\
300\end{array}$ & $98 \mathrm{bcd}$ & $99 \mathrm{bcd}$ & $98 \mathrm{cde}$ & $95 \mathrm{~cd}$ & $97 \mathrm{de}$ & $98 \mathrm{bcde}$ \\
\hline Metribuzin & 413 & \multirow{3}{*}{$100 \mathrm{a}$} & \multirow{3}{*}{$100 \mathrm{ab}$} & \multirow{3}{*}{$100 \mathrm{a}$} & \multirow{3}{*}{$100 \mathrm{a}$} & & \\
\hline Imazethapyr & 77 & & & & & $97 \mathrm{de}$ & $98 \mathrm{bcde}$ \\
\hline Flumioxazin & 96 & & & & & & \\
\hline s-metolachlor/metribuzin & 1443 & 99abcd & $99 \mathrm{cde}$ & $98 \mathrm{bcde}$ & 98abcd & $100 \mathrm{ab}$ & $99 b$ \\
\hline Pyroxasulfone/flumioxazin & 160 & $100 \mathrm{ab}$ & $100 \mathrm{abc}$ & $100 \mathrm{ab}$ & $99 \mathrm{abc}$ & $98 \mathrm{bcde}$ & $99 b c$ \\
\hline
\end{tabular}

Means followed by the same letter within a column are not significantly different according to Fisher's pro-

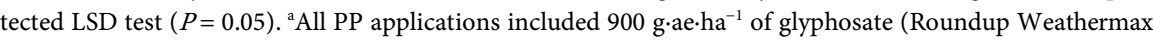
540SL formulation); ${ }^{b}$ All treatments received a POST application of $900 \mathrm{~g} \cdot \mathrm{ae}^{\mathrm{h}} \mathrm{ha}^{-1}$ of glyphosate (Roundup Weathermax 540SL formulation) at the V4 soybean growth stage; ${ }^{\mathrm{C}}$ All data have been pooled across locations and years. Data presented in the table have been backtransformed to the original scale.

Table 7. Soybean seed yield, environmental impact (EI) and partial profit of two-pass weed management strategies used in dicamba-/glyphosate-resistant soybean in 2014 and 2015 in southwestern Ontario.

\begin{tabular}{|c|c|c|c|c|}
\hline \multirow{2}{*}{ Treatment $^{\mathrm{ab}}$} & Rate & Yield $^{c}$ & \multirow{2}{*}{ EI } & Partial Profit \\
\hline & $\mathrm{g} \cdot \mathrm{ai} \cdot \mathrm{ha}^{-1}$ & $\mathrm{t} \cdot \mathrm{ha} \mathrm{a}^{-1}$ & & $\$ h^{-1}$ \\
\hline Weed-Free Control & & $1.82 \mathrm{~b}$ & & \\
\hline Weedy Control & & $2.73 a$ & & \\
\hline Glyphosate & 900 & $2.58 \mathrm{a}$ & 13.8 & $983 a$ \\
\hline Imazethapyr & 100 & $2.67 \mathrm{a}$ & 15.7 & $944 a$ \\
\hline Imazethapyr/saflufenacil & 100 & $2.60 \mathrm{a}$ & 15.2 & $925 a$ \\
\hline Saflufenacil/dimethenamid-p & 245 & $2.66 \mathrm{a}$ & 17.0 & $959 a$ \\
\hline Imazethapyr & 75 & ת & 273 & $0<0_{0}$ \\
\hline Metribuzin & 425 & $2.17 \mathrm{a}$ & 27.3 & $909 a$ \\
\hline
\end{tabular}




\begin{tabular}{ccccc}
\hline Chlorimuron & 9 & $2.73 \mathrm{a}$ & 25.6 & $969 \mathrm{a}$ \\
Metribuzin & 412 & & & \\
Chlorimuron & 9 & $2.63 \mathrm{a}$ & 15.4 & $932 \mathrm{a}$ \\
Imazethapyr & 75 & & & \\
Pyroxasulfone & 100 & $2.75 \mathrm{a}$ & 16.2 & \\
Sulfentrazone & 100 & $2.66 \mathrm{a}$ & 29.6 & $949 \mathrm{a}$ \\
Dicamba & 600 & $2.70 \mathrm{a}$ & 28.2 & $949 \mathrm{a}$ \\
Dimethenamid-p & 544 & & & \\
Dicamba & 300 & $2.68 \mathrm{a}$ & 29.6 & $923 \mathrm{a}$ \\
Metribuzin & 413 & & & \\
Imazethapyr & 77 & $2.73 \mathrm{a}$ & 17.0 & $960 \mathrm{a}$ \\
Flumioxazin & 96 & $2.65 \mathrm{a}$ & 18.0 & $956 \mathrm{a}$ \\
\hline s-metolachlor/metribuzin & 1443 & 160 & & \\
Pyroxasulfone/flumioxazin & & & & \\
\hline
\end{tabular}

Means followed by the same letter within a column are not significantly different according to Fisher's pro-

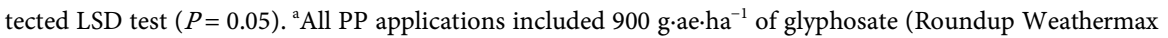
540SL formulation); ${ }^{\mathrm{b}}$ All treatments received a POST application of $900 \mathrm{~g} \cdot \mathrm{ae} \cdot \mathrm{ha}^{-1}$ of glyphosate (Roundup Weathermax 540SL formulation) at the V4 soybean growth stage; 'All data have been pooled across locations and years. Data presented in the table have been backtransformed to the original scale.

United States) spaced $0.5 \mathrm{~m}$ apart. Glyphosate $\left(900 \mathrm{~g} \cdot \mathrm{ae} \cdot \mathrm{ha}^{-1}\right)$ was applied POST at the V4 soybean growth stage. This application represented the second herbicide application timing in the two-pass weed control programs evaluated.

Visual assessments of crop injury were made at 2 and 3 weeks after soybean emergence (WAE) using a scale from 0 to 100 , where 0 represented no injury and 100 indicated soybean death. Six weed species were evaluated including redroot pigweed, common ragweed, common lambsquarters, lady's thumb (Persicaria maculosa S.F. Gray), barnyardgrass (Echinochloa crus-galli (L.) P. Beauv.) and green foxtail (Setaria viridis (L.) P. Beauv.). Weed control was rated visually $3 \mathrm{WAE}$ and before the in-crop POST application of glyphosate; ratings were also conducted at 4 and 8 weeks after POST application (WAA). Weed density and aboveground dry weight were determined for each plot at $3 \mathrm{WAE}$ within a randomly selected $1-\mathrm{m}^{2}$ area. Weeds were placed in paper bags and dried at $60^{\circ} \mathrm{C}$ for 7 days and dry weight was recorded. Soybean seed yield and harvest moisture was determined by harvesting the center two rows of each plot with a small plot combine; yields were adjusted to $13 \%$ moisture.

Treatment effects were assessed by ANOVA using the PROC MIXED procedure in SAS Version 9.4 (SAS Institute, Cary, NC). Data from each experiment were combined across environment and year because no significant interactions between environment and treatment were detected $(P>0.05)$. Random effects included environment, block, and treatment by environment; the fixed effect was the herbicide treatment. The significance of variable and fixed effects were confirmed using the $\mathrm{Z}$ - and F-test at a P-value of $P=0.05$. The assumptions of the ANOVA were confirmed by applying the PROC UNIVARIATE procedure and using the Shapiro-Wilk test for normality together with studentized residual 
plots. Weed control data were transformed before analysis using an arcsine square root transformation while weed density and dry weight received a log transformation. Yield data were not transformed. Means from transformed data were back-transformed for presentation purposes. Treatment means were separated using Fisher's protected LSD at $P=0.05$.

The EI of each herbicide program was calculated using EIQ values from Kovach et al. [29]. The EI was determined by multiplying the EIQ of each active ingredient (ai/ae) by the rate of the ai/ae applied, in $\mathrm{kg} \cdot \mathrm{ha}^{-1}$. To calculate the EIQ of herbicide tank-mixes, the EI of each ai/ae was summed to determine the EI of the herbicide program.

A partial profit analysis compared the profitability of the herbicide treatments. The profitability of each treatment was determined by subtracting the costs of the herbicide and herbicide application from the gross economic return. Gross returns were determined by multiplying the plot yield by the average Ontario soybean price for the month of October in 2014 and 2015 (Blair Andrews, personal communication, 2016). Annual herbicide costs were calculated based on the herbicide retail prices provided by AGRIS (AGRIS Co-operative Ltd., 2015), and the cost of the herbicide application was based on the Ontario Field Crop Budgets [20]. Retail prices for some herbicide programs included in this study were not available, so those products were excluded from the profit analysis. Profitability data were transformed using a square root transformation before analysis.

\section{Results and Discussion}

\subsection{Soybean Injury}

There was minimal soybean injury $(<5 \%)$ observed in this study (data not shown).

\subsection{Weed Control}

There were six dominant weed species in this study including redroot pigweed, common ragweed, common lambsquarters, lady's thumb, barnyardgrass, and green foxtail. Barnyardgrass emerged later in the season so no data are presented for the $3 \mathrm{WAE}$ evaluation timing. There were no weeds at the time of soybean seeding because of secondary tillage before seeding. The glyphosate-only treatment received a POST application of glyphosate (900 g.ae. $\left.\cdot \mathrm{ha}^{-1}\right)$; consequently, the level of weed control was equal to the weedy check prior to the POST application.

Herbicides applied PRE alone or in-combination with others provided good to excellent early season weed control prior to the POST application of glyphosate. At $3 \mathrm{WAE}$, saflufenacil/dimethenamid-p $\left(245 \mathrm{~g} \cdot \mathrm{ai} \cdot \mathrm{ha}^{-1}\right)$ provided the lowest numeric control and controlled redroot pigweed, common ragweed, common lambsquarters, lady's thumb and green foxtail 82, 57, 65, 76 and 57\%, respectively (Table 2). Imazethapyr/saflufenacil (100 g.ai ha $\left.{ }^{-1}\right)$ controlled redroot pig- 
weed, lambsquarters and lady's-thumb $>85 \%$, but provided only $77 \%$ and $69 \%$ control of common ragweed and green foxtail, respectively. Soltani et al. [9] reported similar results where imazethapyr/saflufenacil (100 g.ai.ha $\left.{ }^{-1}\right)$ applied PRE controlled redroot pigweed (98\%) and common lambsquarters (95\%), but had lower control of common ragweed (78\%) and green foxtail (76\%).

Herbicide programs evaluated provided $>80 \%$ control of broadleaved and grass weed species $3 \mathrm{WAE}$ at most study sites, which is similar to the results reported in previous studies evaluating PRE or PP herbicide applications in soybean [9] [10] [11] [12] (Table 2). At 3 WAE, the tank-mix of metribuzin (413

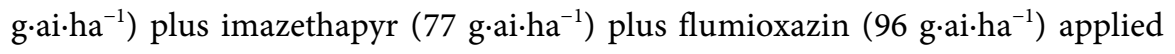
PRE controlled annual broadleaved weeds $>98 \%$ and green foxtail $95 \%$. Imazethapyr (75 g.ai ha $\left.{ }^{-1}\right)$ plus metribuzin $\left(425 \mathrm{~g} \cdot \mathrm{ai} \cdot \mathrm{ha}^{-1}\right)$ and dicamba (600 g.ai $\left.\cdot \mathrm{ha}^{-1}\right)$ applied PRE controlled $>91 \%$ of broadleaved weeds. The results in this study are similar to Soltani et al. [10] who reported imazethapyr $\left(75 \mathrm{~g} \cdot a \mathrm{i} \cdot \mathrm{ha} \mathrm{a}^{-1}\right)$ plus metribuzin (425 g.ai $\cdot \mathrm{ha}^{-1}$ ) controlled common ragweed, redroot pigweed and common lambsquarters $78 \%, 96 \%$ and $99 \%$, respectively [10]. However, the tank-mix

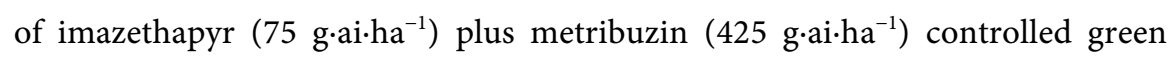
foxtail $91 \%$ and dicamba (600 g.ai $\cdot \mathrm{ha}^{-1}$ ) applied alone provided only $20 \%$ control. The tank-mix of dicamba (300 g.ai $\cdot \mathrm{ha}^{-1}$ ) plus dimethenamid-p (544 $\mathrm{g} \cdot \mathrm{ai} \cdot \mathrm{ha}^{-1}$ ) provided less control of three broadleaved species than the three-way mix of imazethapyr plus metribuzin plus flumioxazin. The tank-mix of dicamba $\left(300 \mathrm{~g} \cdot \mathrm{a} \cdot \mathrm{ha}^{-1}\right.$ ) plus dimethenamid-p (544 g.ai $\cdot \mathrm{ha}^{-1}$ ) controlled green foxtail 90\% compared to $20 \%$ for dicamba applied alone.

\subsection{Density and Dry Weight}

Herbicide tank-mixes applied PRE reduced broadleaf and grass weed density and biomass 3 WAE (Table 3 and Table 4). The application of one or more herbicides reduced the density of redroot pigweed, common ragweed, common lambsquarters, lady's thumb, barnyardgrass, and green foxtail by $92 \%$ to $100 \%$, $25 \%$ to $88 \%, 70 \%$ to $100 \%, 67 \%$ to $100 \%, 27 \%$ to $77 \%$, and $59 \%$ to $98 \%$, respectively (Table 3). Similar trends in weed biomass reduction were also observed

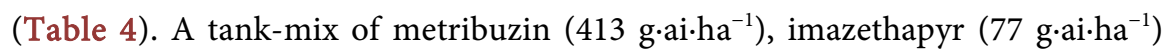
and flumioxazin (96 g.ai $\cdot \mathrm{ha}^{-1}$ ), applied PRE reduced broadleaf weed density and dry weight by $88 \%$ to $100 \%$ and $95 \%$ to $100 \%$, respectively, and reduced grass weed density and dry weight by $59 \%$ to $96 \%$ and $71 \%$ to $96 \%$, respectively. Pyroxasulfone/flumioxazin (160 g.ai.ha $\left.{ }^{-1}\right)$, and imazethapyr $\left(75 \mathrm{~g} \cdot \mathrm{ai} \cdot \mathrm{ha} \mathrm{a}^{-1}\right)$ plus metribuzin $\left(425 \mathrm{~g} \cdot \mathrm{ai} \cdot \mathrm{ha}^{-1}\right)$ reduced weed density and dry weight similar to the previously mentioned three-way tank-mix. Dicamba $\left(600 \mathrm{~g} \cdot \mathrm{ai} \cdot \mathrm{ha}^{-1}\right)$ reduced broadleaved weed biomass and densities $67 \%$ to $92 \%$ and $75 \%$ to $96 \%$, respectively (Table 3 and Table 4). The addition of dimethenamid-p (544 g.ai.ha ${ }^{-1}$ ) to dicamba (300 g.ai $\left.\cdot \mathrm{ha}^{-1}\right)$ improved the control of grass weeds and maintained control of broadleaved weeds.

All PRE followed by glyphosate POST weed management programs pro- 
vided $>95 \%$ weed control 4 WAA where glyphosate $\left(900 \mathrm{~g} \cdot \mathrm{ai} \cdot \mathrm{ha}^{-1}\right.$ ) was applied POST in V4 soybean (Table 5). The tank-mix of metribuzin (413 g-ai.ha ${ }^{-1}$ ), imazethapyr (77 g.ai ha $\left.{ }^{-1}\right)$ and flumioxazin ( $\left.96 \mathrm{~g} \cdot \mathrm{ai}^{\mathrm{h}} \mathrm{ha}^{-1}\right)$ PRE followed by (fb) glyphosate POST provided $100 \%$ control of broadleaf weed species; several herbicide programs provided 99 to $100 \%$ control of broadleaf species following the POST application of glyphosate ( $900 \mathrm{~g} \cdot \mathrm{ai} \cdot \mathrm{ha} \mathrm{a}^{-1}$ ). Glyphosate is a broad spectrum, systemic herbicide that provided excellent control of all weed species evaluated in this study. Several studies reported that a two-pass weed control program of a PRE residual herbicide fb glyphosate POST provided $>88 \%$ full-season weed control [10] [12] [14] [30].

The two-pass weed control programs controlled annual broadleaf and grass weeds similarly at 4 and 8 WAA. Across all treatments, the control of broadleaved and grass was $95 \%$ to $100 \%$ and $94 \%$ to $99 \%$, respectively (Table 6). Although glyphosate does not provide residual weed control [13] [31], it controlled emerged weeds at the time of application, after which the soybean crop canopied over creating an unsuitable environment for weed growth and development [32] [33] [34].

\subsection{Soybean Yield}

Weed interference in the weedy control reduced soybean yield 33\% compared to the weed-free control (Table 7). Reduced weed interference with dicamba (600 g.ai ha $\left.{ }^{-1}\right)$ or dicamba $\left(300 \mathrm{~g} \cdot a \mathrm{i} \cdot \mathrm{ha}^{-1}\right)$ plus dimethenamid-p $\left(544 \mathrm{~g} \cdot \mathrm{a} \cdot \mathrm{ha}^{-1}\right)$ resulted in an increase in soybean seed yield of $46 \%-48 \%$ compared to the weedy control. Soybean yields were similar across weed management programs $(P>0.05$; Table 7); seed yields were $95 \%$ to $100 \%$ of the weed-free control. These findings are consistent with those reported by Soltani et al. [10], Stewart et al. [12], and Van Gessel et al. [35] who found similar soybean yields across two-pass weed control programs of a PP or PRE herbicide fb glyphosate POST.

\subsection{Partial Profit Analysis}

There was no difference in partial profit among herbicide programs. All herbicide treatments produced similar partial profits. Glyphosate was the lowest cost weed management program and was $33 \%$ of the cost of the second lowest cost weed management program. The low cost of glyphosate combined with only one application cost contributes to why this treatment demonstrated the highest partial profit in this study. It is important to note, however, that if a single POST application of glyphosate was not applied in a timely matter, soybean would be exposed to a longer period of early-season weed interference, potentially causing greater yield losses which may result in lower partial profits. Additionally, in fields where higher weed pressures exist, or where one or more difficult-to-control or GR weeds are present, glyphosate may not adequately control the weed population, which could also contribute to greater yield losses and lower partial profits. The development of glyphosate resistant weeds is a topic of 
concern; from a stewardship perspective, a herbicide program consisting only of glyphosate should be avoided to reduce the risk of selecting for GR weed biotypes. Since there were no differences in partial profits between the glyphosate-only herbicide program and other two-pass weed control programs with multiple modes-of-action, it is recommended that a two-pass weed control program be employed.

\subsection{Environmental Impact}

The environmental impact of the herbicide programs included in this study ranged from 13.8 to 29.6 (Table 7). These EI values fall in the range of low or medium environmental risk [12] [15]. Several herbicides that included multiple active ingredients or modes-of-action had only a slightly higher EI than glyphosate applied alone (900 g.ae $\cdot \mathrm{ha}^{-1}$ ); glyphosate (900 g.ae $\cdot \mathrm{ha}^{-1}$ ) applied POST had an EI of 13.8 while the imazethapyr/saflufenacil (100 g.ai.ha $\left.{ }^{-1}\right)$ premix PRE fb glyphosate $\left(900 \mathrm{~g} \cdot \mathrm{ae}^{\mathrm{h}} \mathrm{ha}^{-1}\right)$ POST had an EI of 15.2, chlorimuron $\left(9 \mathrm{~g} \cdot \mathrm{ai} \cdot \mathrm{ha}^{-1}\right)$ plus imazethapyr $\left(75 \mathrm{~g} \cdot \mathrm{ai} \cdot \mathrm{ha} \mathrm{a}^{-1}\right) \mathrm{fb}$ glyphosate $\left(900 \mathrm{~g} \cdot \mathrm{ae} \cdot \mathrm{ha}^{-1}\right)$ POST had an EI of 15.4, and imazethapyr (100 g.ai.ha $\left.{ }^{-1}\right)$ fb glyphosate $\left(900 \mathrm{~g} \cdot a \mathrm{a} \cdot \mathrm{ha}^{-1}\right.$ ) POST had an EI of 15.7. Although a single application of glyphosate had the lowest EI, there is not a substantial increase in the EI with the two-pass weed management programs. By including multiple herbicides and application timings, the diversity of weeds controlled could be increased, the level of weed control early in the growing season could be improved, and the selection of GR weed biotypes could be reduced.

\section{Conclusions}

Generally, two-pass weed control programs provided excellent grass and broadleaved weed control and controlled weeds early in the growing season during the CWFP. Although there were differences in early-season weed control among the herbicide treatments, weeds were controlled by the follow-up POST application of glyphosate. Although past research demonstrates the importance of early weed control during the CWFP, differences in soybean seed yield were not detected. Partial profits were similar among the herbicide treatments. Because of the similarities in yield and partial profit among the treatments, soybean producers may find it attractive to use a single application of glyphosate as their weed control program of choice; however, there are several risks of utilizing a single mode-of-action. Dicamba provided excellent broadleaf weed control, and when combined with a grass herbicide, grass weeds were also controlled. Herbicide programs that contained dicamba resulted in soybean yields and partial profits similar to current industry standards. The use of dicamba in DR soybean will provide control of troublesome broadleaf weeds in soybean, and its importance as a soybean herbicide will increase as the presence of GR broadleaf weeds increases across Ontario.

\section{References}

[1] Shurtleff, W. and Aoyagi, A. (2010) History of Soybeans and Soyfoods in Canada 
(1831-2010): Extensively Annotated Bibliography and Sourcebook. Soyinfo Centre. 2010. http://www.soyinfocenter.com/pdf/137/Cana.pdf

[2] Kulasekera, K. (2016) Estimated Area, Yield, Production, and Farm Value of Specified Field Crops, Ontario, 2012-2016. Online. Ontario Ministry of Agriculture, Food and Rural Affairs.

http://www.omafra.gov.on.ca/english/stats/crops/estimate_new.htm

[3] DeVore, J.D., Norsworthy, J.K. and Brye, K.R. (2013) Influence of Deep Tillage, a Rye Cover Crop, and Various Soybean Production Systems on Palmer Amaranth Emergence in Soybean. Weed Technology, 27, 263-270. https://doi.org/10.1614/WT-D-12-00125.1

[4] Halford, C., Hamill, A.S., Zhang, J. and Doucet, C. (2001) Critical Period of Weed Control in No-Till Soybean (Glycine max) and Corn (Zea mays). Weed Technology, $15,737-744$. https://doi.org/10.1614/0890-037X(2001)015[0737:CPOWCI]2.0.CO;2

[5] Knezevic, S.Z., Evans, S.P. and Mainz, M. (2003) Row Spacing Influences Critical Timing for Weed Removal in Soybean (Glycine max). Weed Technology, 17, 666-673. https://doi.org/10.1614/WT02-49

[6] Mulugeta, D. and Boerboom, C.M. (2000) Critical Time of Weed Removal in Glyphosate-Resistant Glycine max. Weed Science, 48, 35-42. https://doi.org/10.1614/0043-1745(2000)048[0035:CTOWRI]2.0.CO;2

[7] Van Acker, R.C., Swanton, C.J. and Weise, S.F. (1993) The Critical Period of Weed Control in Soybean [Glycine $\max ($ L.) Merr.]. Weed Science, 41, 194-200.

[8] OMAFRA-Ontario Ministry of Agriculture, Food, and Rural Affairs. (2016) Guide to Weed Control. OMAFRA Publ., Toronto, ON, 220-222.

[9] Krausz, R.F., Young, B.G., Kapusta, G. and Matthew, J.L. (2001) Influence of Weed Competition and Herbicides on Glyphosate-Resistant Soybean (Glycine max). Weed Technology, 15, 530-534. https://doi.org/10.1614/0890-037X(2001)015[0530:IOWCAH]2.0.CO;2

[10] Soltani, N., Nurse, R.E. and Sikkema, P.H. (2014) Two-Pass Weed Management with Preemergence and Postemergence Herbicides in Glyphosate-Resistant Soybean. Agricultural Sciences, 5, 504-512. https://doi.org/10.4236/as.2014.56052

[11] Gonzini, L.C., Hart, S.E. and Wax, L.M. (1999) Herbicide Combinations for Weed Management in Glyphosate-Resistant Soybean (Glycine max). Weed Technology, 13, 354-360.

[12] Stewart, C.L., Nurse, R.E., Van Eerd, L.L., Vyn, R.J. and Sikkema, P.H. (2011) Weed Control, Environmental Impact, and Economics of Weed Management Strategies in Glyphosate-Resistant Soybean. Weed Technology, 25, 535-541. https://doi.org/10.1614/WT-D-10-00116.1

[13] Nurse, R.E., Swanton, C.J., Tardif, F. and Sikkema, P.H. (2006) Weed Control and Yield Are Improved When Glyphosate Is Preceded by a Residual Herbicide in Glyphosate-Tolerant Maize (Zea mays). Crop Protection, 25, 1174-1197. https://doi.org/10.1016/j.cropro.2006.02.015

[14] Soltani, N., Stewart, C.L., Nurse, R.E., Van Eerd, L.L., Vyn, R.J. and Sikkema, P.H. (2012) Weed Control, Environmental Impact, and Profitability of Weed Management Strategies in Glyphosate-Resistant Corn. American Journal of Plant Sciences, 3, 1594-1607. https://doi.org/10.4236/ajps.2012.311193

[15] Beckie, H.J. (2006) Herbicide-Resistant Weeds: Management Tactics and Practices. Weed Technology, 20, 793-814. https://doi.org/10.1614/WT-05-084R1.1 
[16] Green, J.M. and Owen, M.D.K. (2011) Herbicide-Resistant Crops: Utilities and Limitations for Herbicide-Resistant Weed Management. Journal of Agricultural and Food Chemistry, 59, 5819-5829. https://doi.org/10.1021/jf101286h

[17] BASF, Canada Inc. (1995) Product Label-Banvel II. BASF Canada Inc., Mississauga.

[18] Heap, I. (2007) The International Survey of Herbicide Resistant Weeds. http://www.weedscience.com

[19] Norsworthy, J.K., McClelland, M. and Griffith, G.M. (2009) Conyza Canadensis (L.) Cronquist Response to Pre-Plant Application of Residual Herbicides in Cotton (Gossypium hirsutum L.). Crop Protection, 28, 62-67. https://doi.org/10.1016/j.cropro.2008.08.012

[20] OMAFRA Ontario Ministry of Agriculture, Food, and Rural Affairs (2016) Field Crop Budgets. OMAFRA Publ., Toronto, 60. http://www.omafra.gov.on.ca/english/busdev/facts/pub60.pdf

[21] Byker, H.P., Soltani, N., Robinson, D.E., Tardif, F.J., Lawton, M.B. and Sikkema, P.H. (2013) Control of Glyphosate-Resistant Horseweed (Conyza canadensis) with Dicamba Applied Preplant and Postemergence in Dicamba-Resistant Soybean. Weed Technology, 27, 492-496. https://doi.org/10.1614/WT-D-13-00023.1

[22] Johnson, B., Young, B., Matthews, J., Marquardt, P., Slack, C., Bradley, K., York, A., Culpepper, S., Hager, A., Al-Khatib, K., Steckel, L., Moechnig, M., Loux, M., Bernards, M. and Smeda, R. (2010) Weed Control in Dicamba-Resistant Soybeans.

https://doi.org/10.1094/CM-2010-0920-01-RS

[23] Spaunhorst, D.J., Siefert-Higgins, S. and Bradley, K.W. (2014) Glyphosate-Resistant Giant Ragweed (Ambrosia trifida) and Waterhemp (Amaranthus rudis) Management in Dicamba-Resistant Soybean (Glycine max). Weed Technology, 28, 131-141. https://doi.org/10.1614/WT-D-13-00091.1

[24] Vink, J.P., Soltani, N., Robinson, D.E., Tardif, F.J., Lawton, M.B. and Sikkema, P.H. (2012) Glyphosate-Resistant Giant Ragweed (Ambrosia trifida L.) Control in Dicamba-Tolerant Soybean. Weed Technology, 26, 422-428. https://doi.org/10.1614/WT-D-11-00184.1

[25] Brookes, G. and Barfoot, P. (2015) Global Income and Production Impacts of Using GM Crop Technology 1996-2013. GM Crops and Food, 6, 13-46. https://doi.org/10.1080/21645698.2015.1022310

[26] Gianessi, L.P. (2005) Economic and Herbicide Use Impacts of Glyphosate-Resistant Crops. Pest Management Science, 61, 241-245. https://doi.org/10.1002/ps.1013

[27] Brimner, T.A., Gallivan, G.J. and Stephenson, G.R. (2005) Influence of Herbicide-Resistant Canola on the Environmental Impact of Weed Management. Pest Management Science, 61, 47-52. https://doi.org/10.1002/ps.967

[28] Fernandez-Cornejo, J. (1998) Environmental and Economic Consequences of Technology Adoption: IPM in Viticulture. Agricultural Economics, 18, 145-155. https://doi.org/10.1016/S0169-5150(97)00054-6

[29] Kovach, J., Petzoldt, C., Degnil, J. and Tette, J.A. (1992) Method to Measure the Environmental Impact of Pesticides. New York's Food and Life Sciences Bulletin, 139, 139-146.

[30] Nurse, R.E., Hamill, A.S., Swanton, C.J., Tardif, F., Deen, W. and Sikkema, P.H. (2007) Is the Application of a Residual Herbicide Required Prior to Glyphosate Application in No-Till Glyphosate-Tolerant Soybean (Glycine max)? Crop Protection, 26, 484-489. https://doi.org/10.1016/j.cropro.2006.04.018 
[31] Payne, S.A. and Oliver, L.R. (2000) Weed Control Programs in Drilled Glyphosate-Resistant Soybean. Weed Technology, 14, 413-422. https://doi.org/10.1614/0890-037X(2000)014[0413:WCPIDG]2.0.CO;2

[32] Bertram, M.G. and Pedersen, P. (2004) Adjusting Management Practices using Glyphosate-Resistant Soybean Cultivars. Agronomy Journal, 96, 462-468. https://doi.org/10.2134/agronj2004.4620

[33] Fedoruk, L.K., Johnson, E.N. and Shirtliffe, S.J. (2011) The Critical Period of Weed Control for Lentil in Western Canada. Weed Science, 59, 517-526.

https://doi.org/10.1614/WS-D-11-00051.1

[34] Norsworthy, J.K. and Oliviera, M.J. (2004) Comparison of the Critical Period for Weed Control in Wide- and Narrow-Row Corn. Weed Science, 52, 802-807. https://doi.org/10.1614/WS-03-165R

[35] VanGessel, M.J., Ayeni, A.O. and Majek, B.A. (2001) Glyphosate in Full-Season No-Till Glyphosate-Resistant Soybean: Role of Preplant Applications and Residual Herbicides. Weed Technology, 15, 714-724. https://doi.org/10.1614/0890-037X(2001)015[0714:GIFSNT]2.0.CO;2 